How to reference this article Nowakowska, M. (2019). Come si traduce in polacco la perifrasi verbale stare per + INFINITO. Italica Wratislaviensia, 10(1), 155-177.

DOI: http://dx.doi.org/10.15804/IW.2019.10.1.7

Małgorzata Nowakowska

Uniwersytet Pedagogiczny w Krakowie

malgorzata.nowakowska@up.krakow.pl, ORCID: 0000-0003-4538-6376

\title{
COME SI TRADUCE IN POLACCO \\ LA PERIFRASI VERBALE STARE PER + INFINITO
}

\section{POLISH TRANSLATIONS OF THE ITALIAN VERBAL CONSTRUCTION STARE PER + INFINITIVE}

\begin{abstract}
The Italian verbal construction stare per + INFINITIVE expresses the prospective aspect. This aspectual meaning can be defined in terms of Reichenbach's theory (1947), which makes use of three points: $\mathrm{E}$ (the point of the event), $\mathrm{S}$ (the point of speech), and $\mathrm{R}$ (the point of reference). The prospective meaning appears when $\mathrm{R}$ precedes $\mathrm{E}$ and when the position of $\mathrm{S}$ is not important. The author of the article examines the possible ways of translating the Italian construction stare per + INFINITIVE into Polish. Unlike Italian, this Slavic language does not have special grams that convey the prospective meaning. Amongst the possible Polish translations, the construction <mieć ('have') + INFINITIVE $>$ seems to express best the prospective meaning of the Italian stare per + INFINITIVE. Still, this Polish construction has two other readings: a modal one and an evidential one.
\end{abstract}

Keywords: prospective aspect, posterior aspect, Italian, Polish, stare per + INFINITIVE, mieć + INFINITIVE 


\section{INTRODUZIONE}

Come sappiamo, ogni lingua categorizza il significato lessicale e grammaticale in modo originale. Questo fatto crea ostacoli a chi fa traduzioni e a chi impara o insegna una lingua straniera. Nella linguistica contrastiva e tipologica si parte spesso da un concetto considerato universale, ossia un tertium comparationis, per poter trovare lessemi o grammemi in due o più lingue esaminate che convoglino lo stesso concetto. Il problema è che non si tratta quasi mai di una forma che esprime lo stesso concetto. Nel caso del significato grammaticale, un grammema con un dato significato in una lingua corrisponde raramente a un grammema in un'altra lingua. Succede spesso che, oltre al significato ricercato, il potenziale corrispondente amalgami un altro significato. Succede anche che non si trovi nessun corrispondente. Spesso, una lingua rende un certo significato non con un grammema ma con un costrutto, vale a dire con una perifrasi o locuzione legata strettamente a un contesto specifico.

Questo articolo tratterà della ricerca di un significato grammaticale, quello della perifrasi verbale stare per, presente nel sistema dell'italiano e assente in quello polacco. Siccome questo significato riguarda la categoria dell'aspetto, comincerò con la sua definizione e poi passerò al significato della perifrasi verbale stare per.

\section{DEFINIZIONE DELL'ASPETTO VERBALE}

Generalmente parlando, si definisce l'aspetto come la maniera in cui il parlante rappresenta la situazione ${ }^{2}$ espressa dal verbo. Questa rappresentazione fornisce indicazioni sulla durata, sul tipo di svolgimento e sul grado di compiutezza della situazione espressa dal verbo. Le informazioni aspettuali possono esser fornite sia dal lessema verbale (azionalità,

1 I miei ringraziamenti vanno a Sebastiano Scarpel per la revisione linguistica di questo articolo.

2 Per situazione intendo azione, processo, evento, attività o stato. È un termine generico che indica qualsiasi tipo di processo espresso dal verbo. 
ossia aspetto lessicale) sia da grammemi aspettuali e temporali (aspetto grammaticale).

Anche se non si può fare un'analisi aspettuale senza prendere in conto l'aspetto lessicale, visto l'argomento trattato nel presente articolo mi limiterò all'aspetto grammaticale. Come vedremo, la descrizione della perifrasi stare per si adatta bene alla definizione dell'aspetto grammaticale in termini di "cinque fasi" (Coseriu, 19803; Dik, 1989). Diverse lingue dispongono di grammemi o di perifrasi verbali che indichino una delle cinque fasi possibili di una situazione. Siccome ogni situazione ha un inizio, uno sviluppo e una fine, la si divide in tre fasi rispettive: iniziale, centrale e terminale. Questa definizione prevede la possibilità di indicare l'intervallo che precede l'inizio della situazione (fase preparatoria) e quello che ha luogo dopo la fine della situazione (fase post-terminativa). Certi linguisti usano il termine di fase eventiva (fr. phase processif in Apothéloz, 2017) per indicare l'intervallo coperto dalla situazione per distinguerla dalle due fasi fuoriuscenti dalla situazione (fase preparatoria e fase post-terminativa). Lo schema citato sotto rappresenta l'aspetto definito come indicazione di una delle cinque fasi in modo grafico.

SCHEMA 1: Cinque fasi di una situazione

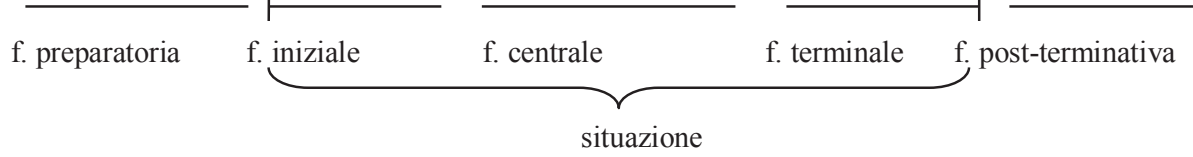

Illustrerò brevemente le cinque fasi con esempi presi dall'italiano perché il suo sistema aspettuale e temporale, contrariamente a quello del polacco, lo permette disponendo di grammemi o perifrasi verbali specifici (cf. Bertinetto, 1990; Squartini, 1998; Strudsholm, 1999).

La fase preparatoria è espressa dalla perifrasi stare per + INFINITO e anche da locuzioni verbali cristallizzate come accingersi a / avviarsi

3 Coseriu e Dik hanno usato diverse denominazioni delle fasi marcate nello schema (1). Inoltre, Coseriu ha distinto due sottotipi della fase iniziale ("ingressiva" e "incettiva") e due fasi della fase terminativa ("conclusiva e "regressiva"). 
$a+$ INFINITO, essere in procinto di + INFINITO, essere sul punto di lè li (li) per + INFINITO (cf. aspetto imminenziale in Bertinetto, 1991) . $^{4} \mathrm{La}$ fase iniziale è resa da cosiddetti "verbi fasali" seguiti dall'infinito come mettersi a, incominciare a, iniziare a (cf. aspetto incoativo o ingressi$v o$ in Bertinetto, 1991). La fase centrale significa la prosecuzione neutrale della situazione oppure la prosecuzione della situazione che mette l'accento sul suo sviluppo o progresso. Il primo caso, chiamato da Bertinetto "aspetto continuativo", è illustrato da locuzioni verbali come continuare $a+$ INFINITO, seguitare $a+$ INFINITO, andare avanti $a+$ INFINITO, mentre il secondo, chiamato "aspetto progressivo", è illustrato da stare + GERUNDIO oppure stare $a+$ INFINITO. La fase terminativa è indicata dai cosiddetti "verbi fasali" come finire di + INFINITO, smetter(la) $d i$ oppure piantar(la) $d i+$ INFINITO. Per parlare di questa fase si usano diversi termini: "aspetto terminativo", "aspetto conclusivo" o "aspetto egressivo". La fase post-terminativa è convogliata da verbi coniugati nei tempi composti: essi non si limitano a esprimere una situazione, ma ne indicano lo stato che ne risulta ${ }^{5}$. Ecco perché si parla dei tempi composti in termini di "aspetto risultativo" o di perfetto ${ }^{6}$.

Per quanto riguarda l'indicazione di una delle cinque fasi, il sistema verbale e aspettuale polacco dispone di molti meno grammemi dell'italiano. È vero che, come molte altre lingue, anche il polacco possiede verbi fasali come zaczać (cominciare), skończyć (finire), o przestać

${ }^{4}$ Per l'inglese, Dik (1989, p. 190) distingue due sottotipi della fase preparatoria: l'aspetto prospettivo (John is going to cry) e l'aspetto prospettivo immediato (John is about to cry). Come vediamo, il primo è reso con la perifrasi to be going to e il secondo, con la perifrasi to bo about to.

${ }^{5}$ Per l'inglese, Dik (1989, p. 190) distingue due sottotipi della fase post-terminativa: Perfect (John has cried) e recent Perfect (John just has cried). Gli esempi citati da Dik fanno pensare che il significato chiamato "recent Perfect" sia dovuto alla presenza dell'avverbio just, senza il quale ci sarebbe l'aspetto chiamato "perfect" (cf. per il francese, Gosselin, 2011).

${ }^{6}$ I tempi composti hanno ereditato il valore risultativo dal latino, anche se nel latino classico il perfectum era una forma semplice. Le forme composte vengono da costruzioni perifrastiche romanze basate su quelle latine come habeo epistulam scriptam (cf. Renzi, 1987, pp. 147-152). È necessario aggiungere che secondo una tradizione terminologica si usa il termine perfetto anche per il passato remoto (ivi, p. 195). 
(smettere), ma non ha un grammema che possa indicare la fase preparatoria né quella post-terminale. L'opposizione aspettuale che si è grammaticalizzata in polacco è quella di perfettività $v s$ imperfettività. Questi due valori aspettuali sono visibili nello schema (1) solamente in modo indiretto. La perfettività è definita come la rappresentazione di una situazione che comporta non solo la fase centrale ma anche quella iniziale e terminativa. Invece l'imperfettività ${ }^{7}$ è definita come la rappresentazione di una situazione che non include né la fase iniziale né quella terminativa. Queste definizioni dicono, in altre parole, che la perfettività dà alla situazione una rappresentazione "totale" o "esterna" (la situazione diventa indivisibile) e che l'imperfettività non dà alla situazione la stessa rappresentazione, ma piuttosto una rappresentazione "interna".

\section{ASPETTO E TEMPO - DUE CATEGORIE GRAMMATICALI}

L'italiano, come numerose lingue, sfrutta grammemi di tempo verbale per convogliare diversi valori aspettuali. Quindi, uno stesso grammema può combinare un valore aspettuale con quello temporale. Al fine di far capire in modo preciso la mia analisi e non confondere la categoria dell'aspetto con quella del tempo, mi soffermerò sulla definizione di tempo verbale. Come leggiamo in grammatiche dell'italiano (cf. ad es. Bertinetto, 1991; Salvi \& Vanelli, 2004; Dardano \& Trifone 2009), il tempo verbale indica il rapporto cronologico che intercorre tra la situazione espressa dal verbo e il momento in cui viene proferito l'enunciato, cioè il momento dell'enunciazione (ME). Questo momento permette di indicare tre possibilità: l'avvenimento accade prima del ME (epoca passata), simultaneamente al ME (epoca presente) o dopo il ME (epoca futura $)^{8}$. I tre schemi messi sotto rappresentano rispettivamente queste

7 Generalmente, si considera l'aspetto iterativo o abituale un sottotipo dell'aspetto imperfettivo, ma lo schema (1) non permette di visualizzarlo.

8 Si noti che Reichenbach (1947) ha definito le tre epoche temporali in modo diverso. Per Reichenbach si trattava del rapporto tra the point of speech (il momento dell'enunciazione) e the point of reference (il momento di riferimento). Però questa inadeguatezza non influisce sul ragionamento presentato in questo articolo, anche perché the point of reference è un termine ambiguo (cf. Apothéloz, 2017). 
tre epoche. Seguendo la tradizione terminologica ispirata a Reichenbach (1947), uso i termini "momento dell'enunciazione" (ME) e "momento dell'avvenimento" (MA), anche se non si tratta veramente di momenti ma di intervalli temporali di una certa lunghezza. È questo il motivo per il quale i "momenti" sono resi graficamente non con un punto ma con un segmento di linea.

Schema 2: Il passato

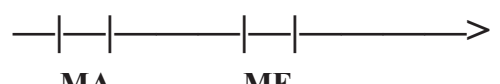

MA ME

Schema 3: Il presente



SCHEMA 4: Il futuro

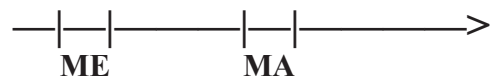

\section{ASPETTO PROSPETTIVO ESPRESSO DALLA PERIFRASI STARE PER}

Per denominare l'aspetto della perifrasi verbale stare per + INFINITO e di altre perifrasi come $<$ accingersi a / essere sul punto di / essere in procinto $d i+$ INFINITO>, Bertinetto (1991) usa il termine "aspetto imminenziale". A questo termine preferisco "aspetto prospettivo"10, che ha un significa-

${ }^{9}$ Cf. Coseriu (1980), che chiama questa fase "imminenziale" (fr. imminentiel) (cf. Jansen, 2010).

${ }_{10}$ Benveniste (1959) usa il termine prospectif per denominare l'aspetto del tempo francese chiamato "futur proche" ossia "futur périphrastique", tempo verbale che si 
to meno specifico, ossia non evoca il carattere prossimo e immancabile della situazione espressa dal verbo ${ }^{11}$.

Come abbiamo già accennato, si può definire l'aspetto espresso dalla perifrasi stare per, ossia l'aspetto prospettivo, come l'indicazione della fase preparatoria. Tuttavia, questa definizione non sembra sufficiente per render conto del suo vero significato. Mi sembra più precisa la definizione basata sulla teoria di Reichenbach (1947). A questo scopo userò i suoi tre punti: momento dell'avvenimento MA, quello dell'enunciazione $\mathrm{ME}$ e quello di riferimento $\mathrm{MR}^{12}$.

Per definire l'aspetto prospettivo sono importanti due fattori: il primo è che l'avvenimento è separato dal punto di riferimento (come se si indicassero due "temporalità" alla volta); il secondo è che il MR precede il MA. In altre parole, l'avvenimento espresso dal verbo è considerato da un punto di riferimento che lo precede, il che dà all'avvenimento una visione "prospettiva". Reichenbach (1947, p. 297) marca la noncoincidenza dei momenti (ingl. points) con un tratto (-) e la coincidenza, con una virgola (,). In questo modo, nello schema della prospettività, si ripete sempre la sequenza MR-MA. Inoltre, Reichenbach situa questa combinazione in tre epoche: presente (posterior present), passata (posterior past) e futura (posterior future). Le tre epoche sono indicate tramite il rapporto tra ME e MR, come vediamo sotto:

(1a) posterior present

(1b) posterior past

(1c) posterior future
ME,MR-MA

MR-MA-ME / MR-MA,ME / MR-ME-MA

ME-MR-MA

può definire usando una combinazione dei tre punti di Reichenbach, come faccio in questo articolo per la perifrasi verbale stare per. Va aggiunto che la perifrasi $<$ dovere + INFINITO> con significato di "futuro nel passato" è chiamata da Bertinetto "perifrasi prospettiva" (1991, p. 160).

11 Come vedremo in seguito, la perifrasi stare per non implica la realizzazione della situazione espressa dal verbo all'infinito, e ciò mette in discussione il carattere 'immancabile' del significato di stare per.

12 Qui si adottano i termini italiani. Il momento dell'avvenimento corrisponde a $\mathrm{E}$ (point of the event), quello dell'enunciazione a $\mathrm{S}$ (point of speech) e quello di riferimento a $\mathrm{R}$ (point of reference). 
Si noti che Reichenbach prevede tre combinazioni per la prospettività nel passato a seconda della posizione del momento dell'avvenimento MA: anteriore al ME, concomitante con il ME e posteriore al ME. Nel caso della perifrasi stare per si tratta solo della prima combinazione: $M R-M A-M E$. Ovviamente, oltre che all'epoca passata, questa perifrasi si applica al presente: $M E, M R-M A$. Non è applicata all'epoca futura, visto che non appare con il verbo fraseologico ${ }^{13}$ al futuro.

Seguendo la simbolizzazione della prospettività di Reichenbach, propongo gli schemi grafici di un esempio con stare per: l'uno con il verbo fraseologico al presente e l'altro, all'imperfetto ${ }^{14}$.

Schema 5: Aspetto prospettivo (sto per uscire) ${ }^{15}$

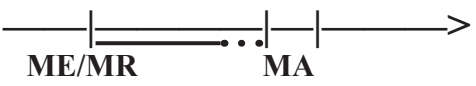

SCHEMA 6: Aspetto prospettivo (stavo per uscire)

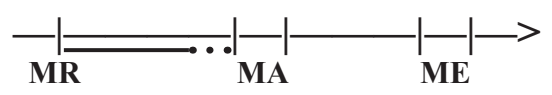

Questi schemi mettono in evidenza due fattori: che la fase preparatoria copre l'intervallo tra il MR e il MA e che l'uso della perifrasi stare per non implica il raggiungimento dell'avvenimento, il che è stato marcato con una linea tratteggiata.

${ }_{13}$ Il verbo semi-ausiliare stare è chiamato "verbo fraseologico" e il verbo all'infinito introdotto dalla preposizione, "verbo lessicale" o "verbo nucleare" (cf. Jansen, 2010). La terminologia adottata da Bertinetto (1990) è diversa: il verbo semi-ausiliare coniugato ai tempi verbali finiti è chiamato "modificatore" mentre il verbo alla forma verbale indefinita è chiamato "modificato" o "principale".

${ }^{14}$ Cf. Bertinetto (1991, pp. 158-159) per le restrizioni d'uso della perifrasi stare per.

15 Questo schema semplifica la relazione tra il ME e il MR. Il ME dovrebbe includere il MR se accettiamo l'idea di Apothéloz (2017) che il MR è piuttosto un punto e non un segmento di linea, contrariamente al ME e MA. In questa prospettiva il MR non costituisce una referenza temporale ma un punto di riferimento. 


\section{TRADUZIONE IN POLACCO DELLA PERIFRASI VERBALE $<$ STARE PER + INFINITO $>$}

Come è già stato accennato, il polacco non dispone di mezzi grammaticali che indichino la fase preparatoria. Quindi la resa di questo significato aspettuale si caratterizzerà per una certa perdita, a meno di trovare in polacco qualche costrutto più o meno cristallizzato. Passerò ora alla presentazione dei principali modi di rendere questo significato (cf. Apothéloz \& Nowakowska, 2016). Le mie osservazioni sono fondate sull'analisi di alcune traduzioni di romanzi italiani in polacco.

\subsection{Locuzioni verbali}

Cercando corrispondenti polacchi della perifrasi stare per, si notano delle locuzioni verbali con la seguente struttura: <verbo fraseologico + preposizione + nome astratto $>$. Fanno parte delle espressioni idiomatiche, essendo sottomesse a diverse restrizioni circa la collocazione dei loro componenti. Innanzitutto, i verbi fraseologici e i nomi astratti che costituiscono un paradigma sono di numero ristretto. I nomi astratti possono essere preceduti dalla preposizione $n a$ o $k u$ a seconda dei nomi. Il verbo fraseologico, che non si può tradurre in italiano a causa del suo svuotamento semantico, ha una forma impersonale, che è, in questo caso, la terza persona singolare. Osserviamo tre verbi fraseologici che si combinano con la preposizione $n a$, preposizione usata originalmente per indicazioni spaziali:

(2) zbiera się na deszcz (pioggia) idzie na burzę (tempesta) zanosi się na awanturę (putiferio)

Tutti questi verbi, nel loro significato originale, denotano un movimento $^{16}$. Come si vede in (2), i nomi astratti denotano situazioni senza controllo del soggetto, ossia non intenzionali. Questa caratteristica ri-

16 Si può completare questo paradigma con il verbo zapowiadać się, che non ha un significato di movimento, ma è affine a preannunciarsi o prospettarsi. Cf. la traduzione Zapowiada sie na bardzo ładny dzień. - Si prospetta proprio una bella giornata. Però, 
guarda anche la locuzione con mieć się (letteralmente aversi) in funzione di verbo fraseologico. Questo verbo, che indica in origine uno stato e non un movimento, si colloca con la preposizione $n a$, come i verbi precedenti, oppure con la preposizione $k u$, che conserva il suo significato 'verso' 17 :

(3) ma się na burzę (tempesta)

świtanie (alba)

dłuższą pogodę (bel tempo durevole)

poprawę (miglioramento)

odmianę (cambiamento), ecc.

(4) ma się $\quad \mathrm{ku}$ lepszemu (il meglio)

wieczorowi (sera)

wiośnie (primavera)

zachodowi (tramonto del sole)

kolacji (cena), ecc.

Osservando i nomi astratti citati sopra, emerge che solo in pochi casi queste espressioni sono delle traduzioni della perifrasi stare per:

(5) Zbiera się na deszcz (pioggia) - Sta per piovere.

Zbiera się na burzę (tempesta) - Sta per...?

Zbiera się na zimę (inverno) - Sta per...?

Questo fatto è dovuto alla mancanza in italiano di verbi corrispondenti ai nomi astratti polacchi. Tranne certi casi come zmiana (cambiamento), neanche in polacco il significato di questo tipo di nomi astratti si lascia esprimere da un verbo. Tutto sommato, queste locuzioni verbali sono specializzate in questo tipo di significati. Va aggiunto che, in certi casi, l'italiano esige la forma personale della perifrasi:

(6) Ma się ku zachodowi (tramonto del sole) - Il sole sta per tramontare.

al contrario del resto dei verbi fraseologici di questo tipo di locuzioni verbali, il verbo zapowiadać się può anche essere seguito da avverbi.

${ }_{17}$ La preposizione $n a$ regge il nome all'accusativo e la preposizione $k u$, il nome al dativo. 
Detto questo, pur non "traducendo" la perifrasi stare per, le locuzioni verbali polacche esprimono uno stesso significato aspettuale, la prospettività. Più precisamente, è il verbo fraseologico combinato con una delle tre preposizioni che la esprime. Questo significato è parafrasabile con 'avvicinarsi a'. Ma, come abbiamo visto, la struttura sintattica delle locuzioni polacche differisce da quella di stare per. Le locuzioni polacche bloccano la posizione di soggetto (hanno una forma impersonale) e il "soggetto logico"18 è dato tramite un sintagma preposizionale con un nome astratto. Come si sa, la struttura di stare per non pone nessuna restrizione sul soggetto o primo argomento, come vediamo in (5) e (6). L'unica eccezione che si conosce riguardo al soggetto nelle locuzioni polacche è la locuzione che si compone del verbo fraseologico zbierać się seguito dalla preposizione $d o$, e non $n a$, e da un nome astratto. Come mostra il seguente esempio abbiamo a che fare con un vero soggetto:

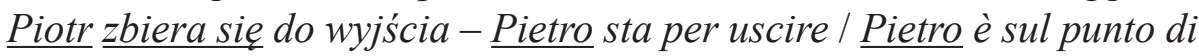
uscire.

\subsection{Costrutto polacco fondato sulla perifrasi $<$ mieć (avere) + INFINITO>}

Esiste una possibilità di rendere assai fedelmente la perifrasi stare per in polacco: si tratta della perifrasi <mieć (avere) + INFINITO> completata semanticamente da un contesto specifico. Il significato fornito dal contesto è necessario visto che questa perifrasi è polisemica. Innanzitutto, ha un significato modale (deontico o aletico), ma ha anche un significato evidenziale (la seconda fonte dell'informazione) (cf. Topolińska, 1968, 2000; Lempp, 1986; Holvoet, 2012). Quindi, perché sia interpretata come 'indicazione della fase preparatoria', deve apparire in un contesto che permetta di annullare gli altri due significati. Proprio per questo motivo uso il termine di costrutto: denomina la collocazione della perifrasi <mieć (avere) + INFINITO > con un contesto specifico. Come ogni costrutto, è molto più libero delle espressioni idiomatiche: permette di sostituire un suo componente con un altro e anche di cancellare uno dei

18 Dal punto di vista sintattico, non è sicuro che un sintagma preposizionale possa svolgere la funzione di soggetto. 
suoi componenti. In questo articolo mi limito a fornire solo due esempi più o meno canonici di questo tipo di costrutto (per gli altri cf. Nowakowska, 2017):

(7a) < stare per + INFINITO ... quando ... (+ verbo con aspetto perfettivo) $>$

(7b) <avverbio już + mieć (avere) + INFINITO ... kiedy ... (+ verbo con aspetto perfettivo)>

Si noti che la traduzione con questo costrutto polacco è possibile solo nelle situazioni in cui il contesto italiano della perifrasi stare per abbia la forma identica a quella di (7a). In altri termini, la perifrasi italiana deve trovarsi nella frase principale seguita dalla temporale (introdotta da quando). Va aggiunto che nel costrutto polacco c'è un componente supplementare: l'avverbio już, che è delessicalizzato in questo uso, ma il cui significato è affine agli avverbi italiani come già o proprio:

(8a) Stavo per andarmene, annoiato da quel cicaleccio, quando udii Salon. Parlava sottovoce, con tensione, come se stesse trattenendo qualcuno per il braccio. Riconobbi la voce di Pierre. (Eco, Il pendolo di Foucault)

(8b) Już miałem odejść, znudzony tą paplaniną, kiedy usłyszałem Salona. Mówił półgłosem, z napięciem, miałem wrażenie, jakby ciągnął kogoś za ramię. Rozpoznałem głos Pierre'a. (Eco, Wahadło Foucaulta, trad. Adam Szymanowski)

Si noti, nella temporale, la presenza del passato remoto udii, che ha valore perfettivo $^{19}$. Questo valore è reso in polacco con il passato perfettivo ustyszatem ${ }^{20}$. Il costrutto polacco (7b), e anche quello italiano (7a), si basano proprio sul contrasto tra una situazione la cui fine non è asserita (indicazione della fase preparatoria) e una situazione data come totale (espressa dal verbo con valore perfettivo). Va ricordato qui lo schema

19 In italiano, questo valore può esser attribuito solo ai grammemi di passato remoto. Se ammettiamo che il passato prossimo nell'uso aoristico non porti alla sparizione del MR, non possiamo attribuirgli un valore perfettivo, come lo definisco in questo articolo (cf. Nowakowska, 2018).

${ }^{20}$ In polacco, contrariamente all'italiano, l'opposizione perfettivo $v s$ imperfettivo è presente non solo nel tempo verbale del passato ma anche in quello del futuro. 
(6), che individua il carattere virtuale della chiusura dell'intervallo che intercorre tra il MR e l'inizio della situazione espressa dal verbo lessicale (il MA). Nell'esempio (8), si può dire che il fatto di udire Salon coincide con l'avvicinarsi all'uscita e non con l'uscita. In questo caso preferisco parlare di simultaneità parziale tra la situazione espressa dalla principale e quella espressa dalla subordinata temporale, invece di dire, come fanno numerosi linguisti, che la seconda ha interrotto la prima. Questa constatazione non è precisa dal momento che la perifrasi stare per non comunica il non-proseguimento della prima situazione. Questo proseguimento o non-proseguimento è un'informazione fornita dal contesto seguente. La relazione temporale tra l'udire e lo stare per andarsene è tratteggiata nel seguente schema, in cui l'udire è rappresentato con un tratto verticale messo sulla prima linea del tempo:

SCHEMA 7: Simultaneità parziale

frase temporale frase principale



L'analisi delineata nello schema (7) è valida anche per la perifrasi polacca $<$ mieć + INFINITO $>$ in (8b). Inoltre, siccome il verbo mieć (avere) non denota altro che uno stato, l'avverbio $j u \dot{z}$ aggiunge alla perifrasi un tratto dinamico che si interpreta qui come progressione verso un termine.

Infine, si noti che questo costrutto, a causa della sua struttura, fa parte della cosiddetta "subordinazione inversa". Questa struttura sintattica era presente nel latino (cum inversum $)^{21}$ e poi, in altre lingue romanze

${ }^{21}$ È interessante scoprire che esiste un parallelismo tra il polacco e il latino per quanto riguarda il "cum inversum". In questo costrutto, anche in latino si nota la presenza di un avverbio che corrisponde al polacco już: si tratta di iam, come vediamo nella seguente frase tradotta letteralmente in italiano: Hannibal iam subibat, Romani erumpunt in eum cum repente. - Annibale si stava già avvicinando, quand'ecco $i$ Romani fecero una sortita contro lui. Inoltre, osserviamo la presenza di quand'ecco, variante della congiunzione temporale che corrisponde a <cum (quando) + repente (all'improvviso) $>$ e sottolinea la velocità ossia la breve durata della situazione espressa 
(cf. Bianco, 2013). Quindi, oltre alla caratteristica temporale e aspettuale, è importante evidenziare l'ordine rovesciato delle proposizioni nella frase temporale. Infatti, in (8ab) la temporale segue la principale. Questo ordine differisce da quello canonico, che vediamo in una frase come "Quando Cesare giunse nella Gallia, devastò ogni cosa". Qui la temporale serve da localizzatore temporale, mentre ciò non vale per le frasi (8ab). In questi esempi la relazione tra la principale e la temporale è di tipo paratattico. La debole dipendenza della temporale dalla principale fa sì che si può staccarle l'una dall'altra e dire ad es. Stavo per andarmene, annoiato da quel cicaleccio e in quel momento udii Salon (cf. Le Draoulec, 2003, 2006) ${ }^{22}$.

Il costrutto che sto esaminando non si limita però alla cosiddetta "subordinazione inversa". Una delle varianti di questo costrutto permette infatti la sostituzione di quando con la congiunzione avversativa ma:

(9a) < stare per + INFINITO ... ma ... (+ verbo con aspetto perfettivo $)>$

(9b) <avverbio już + mieć (avere) + INFINITO ... ale ... (+ verbo con aspetto perfettivo) $>$

Come vedremo nel seguente esempio, questa congiunzione non serve a introdurre un contrasto a livello logico ma aspettuale:

(10a) Mi sedetti su un divano ai cui piedi giaceva una grande coppa di vetro, ancora piena a metà di macedonia. Stavo per prenderne un poco, perché non avevo cenato, ma ebbi l'impressione di scorgervi come lo stampo di un piede, che aveva pressato al centro i cubetti di frutta, riducendoli a un pavé omogeneo. (Eco, Il pendolo di Foucault)

(10b) Usiadłem na kanapce, przy której stał wielki szklany półmisek, jeszcze do połowy wypełniony surówką. Już miałem jej sobie nabrać, bo nie jadłem kolacji, ale wydało mi się, że widzę jakby odcisk stopy,

dalla temporale. Questa situazione è opposta a quella espressa dalla principale che, come si vede nella traduzione italiana, contiene una perifrasi con valore imperfettivo (progressivo).

${ }^{22}$ Un altro dei criteri esaminati da Le Draoulec $(2003,2006)$ è il significato presupposto: contrariamente alla temporale canonica, la subordinazione inversa è esente dal presupposto. 
która stąpnęła w sam środek owocowych kostek, zmieniając je w jednolitą miazgę. (Eco, Wahadło Foucaulta, trad. Adam Szymanowski)

La congiunzione avversativa serve a mettere in rilievo un contrasto: nell'intervallo che precede la situazione 'prendere un poco di macedonia' ne avviene un'altra: 'avere l'impressione di...'. La seconda situazione è espressa con un tempo verbale con valore perfettivo in italiano (passato remoto) e in polacco (passato perfettivo).

\subsection{Verbo modale polacco + INFINITO}

Ci sono traduzioni polacche che rendono meno fedelmente il significato della perifrasi stare per. Lo fanno usando una perifrasi che si compone di un verbo modale volitivo o deontico seguito dall'infinito del verbo lessicale. Con questa traduzione si fuoriesce dalla categoria dell'aspetto. Ricordiamo che, come si vede negli schemi (5) e (6), l'indicazione della fase preparatoria lascia indeterminata l'informazione sul raggiungimento dell'avvenimento (il MA). Questo fattore permette di intenderlo come potenziale. Questa caratteristica può esser considerata comune con il significato modale, visto che lo si definisce come quel significato che mette un enunciato fuori dalle dimensioni "vero" o "falso", cioè nell'ambito della possibilità, della necessità o della volontà (cf. Gosselin, 2011).

\subsubsection{Verbo modale con significato volitivo}

Ovviamente, questa traduzione vale solo per verbi che denotano azioni intenzionali: non è possibile tradurre in questo modo un enunciato come Sta per piovere. Nel primo esempio citato sotto il traduttore impiega il verbo chcieć (volere) e nel secondo, zamierzać (intendere). Tutti e due sono seguiti dal verbo lessicale corrispondente al verbo lessicale italiano:

(11a) Capii subito che lui stava per acchiapparmi le zinne e gli dissi subito, tirandomi indietro: "No [...]". (Moravia, La Ciociara) 
(11b) Domyśliłam się, że chce chwycić mnie za sutki, i cofając się powiedziałam obcesowo: - Nie [...]. (Moravia, Matka i córka, trad. Zofia Ernstowa)

(12a) Visto che stavo per mettere il mio cuore a nudo, come il poeta, quasi senza accorgermene sono passato per istinto al tu. (Eco, Numero zero)

(12b) Ponieważ zamierzałem otworzyć przed nią serce jak poeta, instynktownie, prawie tego nie zauważając, przeszedłem na ty. (Eco, Temat na pierwsza stronę, trad. Krzysztof Żaboklicki)

\subsubsection{Verbo modale con significato deontico}

Si può tradurre la perifrasi stare per con un verbo deontico se il contesto precedente fornisce indizi che lo permettono. In (13a) il personaggio principale del testo di Ciociara (io) deve lasciare la sua casa a causa della guerra. In (14a) si capisce che l'interlocutore è un giornalista poiché ha il compito di dirigere un giornale.

(13a) $[\ldots]$ io invece stetti a occhi aperti pensando tutto il tempo a Giovanni, ai sacchi di carbone e alla manata che lui mi aveva dato sul sedere e alla casa e al negozio che stavo per lasciare. (Moravia, La Ciociara)

(13b) [...] a ja leżąc z otwartymi oczyma myślałam cały czas o Giovannim, o workach z węglem, o uderzeniu po tyłku, o sklepie i mieszkaniu, z którymi musialam się rozstać. (Moravia, Matka i córka, trad. Zofia Ernstowa)

(14a) E vuole che lo scriva io? Perché non lo scrive lei? È un giornalista, no? Almeno, visto che sta per dirigere un giornale... (Eco, Numero zero)

(14b) - I chce pan, żebym to ja ją napisał, a dlaczego nie pan? Jest pan przecież dziennikarzem, w każdym razie ma pan redagować gazetę... (Eco, Temat na pierwsza stronę, trad. Krzysztof Żaboklicki)

In (13b) il traduttore impiega il verbo modale musieć, che ha un significato più preciso di quello del verbo italiano dovere: denota una necessità vista come un obbligo per motivi interni. In (14b) il traduttore impiega il verbo mieć che, come ho già accennato, può avere un significato deontico oltre a quello aspettuale o evidenziale. La perifrasi $<$ mieć 
(avere) + INFINITO> significa qui una necessità "debole", cioè piuttosto 'avere un compito da fare' che 'avere un obbligo'.

$\mathrm{Va}$ aggiunto che il significato modale, che sia volitivo o deontico, non risulta controfattuale. Il vero significato controfattuale implica un fatto che non è avvenuto nel passato come in: Se il vaso fosse caduto, si sarebbe rotto. Questo non avviene per la perifrasi stare per all'imperfetto. Contrariamente all'enunciato di periodo ipotetico appena citato, questa perifrasi non comunica la non-realizzazione della situazione nel passato, ma lascia la sua realizzazione sotto-determinata.

\subsection{Verbo polacco al futuro}

Nella traduzione polacca della perifrasi stare per con un verbo al futuro si perde il significato aspettuale, quello dell'indicazione della fase preparatoria. Paragonando lo schema (4) con (5), viene fuori che il futuro ha un significato più povero rispetto a quello della fase preparatoria: quest'ultima esige un momento di riferimento (MR) in più. Per rappresentare il futuro, invece, bastano due momenti: il momento dell'avvenimento (MA) e il momento dell'enunciazione (ME). In altre parole, manca il collegamento con il momento dell'enunciazione (ME) dell'avvenimento situato dopo questo momento, ossia dopo il ME. Invece, la perifrasi stare per implica questo collegamento: dà all'avvenimento una rappresentazione prospettiva.

(15a) Improvvisamente la sala cadde nella penombra e le pareti si illuminarono. Mi accorsi che erano ricoperte per tre quarti da uno schermo semi-circolare su cui stavano per essere proiettate delle immagini. (Eco, Il pendolo di Foucault)

(15b) Nagle salę ogarnął półmrok, natomiast rozświetliły się ściany. Dopiero teraz zauważyłem, że są w trzech czwartych zakryte półokrągłym ekranem, na który rzucać się będzie obrazy. (Eco, Wahadło Foucaul$t a$, trad. Adam Szymanowski)

Il futuro polacco impiegato nella traduzione ha valore imperfettivo. Si compone del verbo ausiliare być (essere) coniugato al futuro (będzie) seguito dal verbo all'infinito. Il passivo essere proiettate è stato reso da un verbo con il si passivante: rzucać się. Inoltre, in italiano è presente 
la concordanza dei tempi, che manca nella lingua polacca. Perciò nella traduzione c'è il futuro e non "il futuro nel passato".

Come è già stato detto, il significato aspettuale è residuale nella traduzione: rimane solo l'informazione sulle proiezioni localizzate dopo un momento attuato e indicato dal filo narrativo. Possiamo chiederci quanto grande sia il residuo. I linguisti e soprattutto i logici affermano che il futuro non è un vero tempo verbale (cf. Lyons, 1989, § 15.4) perché, contrariamente al passato e al presente, non si sottomette al giudizio "vero" o "falso". Per questo motivo si situa tra due categorie: quella del tempo e quella della modalità. Ciò che lo avvicina alla fase preparatoria è proprio il carattere potenziale della situazione che esprime.

\subsection{Perifrasi <mieć (avere) + INFINITO> con significato evidenziale}

La perifrasi <mieć (avere) + INFINITO> può avere un significato di 'seconda fonte dell'informazione', significato che rappresenta un sottotipo della categoria dell'evidenzialità (cf. Blakemore, 1999). Questo significato è stato scoperto nella perifrasi <mieć (avere) + INFINITO > da linguisti polacchi che si occupano di lingue balcaniche o baltiche che hanno grammaticalizzato questo sottotipo di evidenzialità (Topolińska, 1968, 2000; Holvoet, 2012). Si tratta dell'uso del verbo per significare una certa "distanza" verso il messaggio comunicato. Una forma verbale marca allora che l'informazione è fornita da qualcuno d'altro: in inglese lo si chiama hearsay ('il sentito dire'). Ci sono anche altri termini impiegati per definire questo uso (cf. Jakobson, 1963; Fici, 2008), ma io seguo in questa analisi il termine di mediatività che ha proposto Guentchéva (1994), prima per la descrizione del bulgaro e dopo per le lingue romanze. Analizziamo un esempio in cui la perifrasi stare per è preceduta dal contesto mediativo:

(16a) E si diceva che all'aeroporto di Bresso stava per atterrare un velivolo per prelevare il dittatore. Il Comitato di liberazione era convinto che Mussolini in mano agli alleati se la sarebbe cavata, magari chiuso in una fortezza per qualche anno, e poi sarebbe tornato in scena. Luigi Longo (che nel comitato rappresentava i comunisti) aveva invece detto che Mussolini era da accoppare subito, in malo modo, senza processo e senza frasi storiche. (Eco, Numero zero) 
(16b) Mówiono też, że na lotnisku Mediolan-Bresso ma wylądować samolot, który zabierze dyktatora. Komitet Wyzwolenia był przekonany, że Mussolini z rąk aliantów wyszedłby cało, osadziliby go na pewno w jakiejś twierdzy na kilka lat, a później wróciłby na scenę. Luigi Longo (przedstawiciel komunistów w Komitecie) powiedział zaś, że Mussoliniego należy ukatrupić od razu, w haniebny sposób, bez procesu i historycznych przemów. (Eco, Temat na pierwszą stronę, trad. Krzysztof Żaboklicki)

Il significato mediativo è rappresentato dall'espressione impersonale si diceva. Quindi l'informazione sull'arrivo di Mussolini era fondata su una diceria comune, ossia non attribuita a un locutore particolare. Questo significato è ancora rafforzato dal contesto seguente che segnala una controversia su questa notizia (Luigi Longo (che nel comitato rappresentava i comunisti) aveva invece detto che Mussolini...). Quindi è proprio il contesto mediativo che giustifica la traduzione polacca con la perifrasi <mieć (avere) + INFINITO $>23$.

Come per la traduzione con un verbo al futuro (vedi sopra), anche qui abbiamo a che fare con un residuo: si perde il significato della fase preparatoria. Invece, comunicando una distanza verso la fonte dell'informazione, si può avere per effetto una specie di liberazione del locutore o scrittore della responsabilità circa la verità del messaggio. Quindi, anche il significato mediativo fornisce un dubbio sulla verità o falsità dell'enunciato. Questa caratteristica avvicina la mediatività alla fase preparatoria: tutte e due implicano, almeno in una parte del loro significato, una potenzialità (Squartini, 2003; Gosselin, 2011). Questa analisi non cerca di attribuire alla perifrasi stare per un significato mediativo ${ }^{24}$, per il quale si caratterizza la perifrasi polacca <mieć (avere) + INFINITO>. Cerca solo di evidenziare un significato costruito dalla perifrasi italiana e dal contesto, che porta a questa e non a un'altra traduzione in polacco.

23 Anche in questo esempio si nota l'assenza della concordanza dei tempi in polacco: il verbo fraseologico mieć della perifrasi è coniugato al presente $(\mathrm{ma})$.

24 In italiano si marca il significato mediativo ad esempio con un condizionale detto "giornalistico" o "di riserva". 


\section{OSSERVAZIONI FINALI}

L'italiano è più ricco del polacco per la possibilità di esprimere la fase preparatoria. È la perifrasi stare per che convoglia questo significato. Malgrado questa lacuna nel sistema aspettuale polacco, esistono diversi modi di rendere il significato prospettivo oppure un significato simile. Nella rassegna di questi modi fatta in questo articolo, due traduzioni appaiono come più fedeli: (1) locuzioni verbali fondate su un verbo fraseologico seguito da una preposizione e un nome astratto e (2) un costrutto di tipo <AVVERBIO już + mieć (avere) + INFINITO... kiedy (quando)... (+ VERBO con aspetto perfettivo) $>$. Tutte e due indicano la fase preparatoria ma non possono esser impiegate in certi casi. Le locuzioni verbali polacche con un nome astratto, essendo impersonali, si limitano ad esprimere delle situazioni senza controllo del soggetto. Sono di numero limitato e sono impiegate per esprimere la fase preparatoria in certe situazioni come cambiamenti meteorologici, temporali o altro, a condizione cioè che non ci sia un agente. Il costrutto di tipo <AVVERBIO już + mieć (avere) + INFINITO... kiedy (quando) ... (+ VERBO con aspetto perfettivo) $>$ esige un contesto narrativo specifico, e ciò costituisce una certa restrizione d'uso.

In altri casi, il traduttore è costretto a utilizzare i mezzi che vengono dalla categoria della modalità, del tempo o della mediatività. La prima categoria è rappresentata da verbi modali volitivi o deontici, la seconda dal futuro e la terza dalla perifrasi verbale <mieć (avere) + INFINITO $>$. In tutti questi casi c'è un residuo riguardante l'indicazione della fase preparatoria. Queste traduzioni, però, sfruttano il loro significato potenziale per avvicinarsi a quello della fase preparatoria, la quale, infatti, non implica il raggiungimento all'avvenimento veicolato dal verbo lessicale della perifrasi stare per. È vero che il verbo lessicale indica un avvenimento ma la perifrasi stare per comunica solo l'avvicinamento a questo avvenimento e non la sua realizzazione. Proprio per questo esiste un parallelismo con il significato modale, futurale e mediativo. 


\section{BIBLIOGRAFIA}

Apothéloz, D. (2017). Reichenbach revisité. Verbum, XXXIX (1), 5-30.

Apothéloz, D., \& Nowakowska, M. (2016). Comment traduire le futur périphrastique français en polonais ? In O. Inkova \& A. Trovesi (Eds.), Langues en constrastes / Славянские языки in comparatione / Lingue a confronto (pp. 55-83). Bergamo: Sestante.

Benveniste, E. (1959). Les relations de temps dans le verbe français. Bulletin de la Société de Linguistique LIV, fasc. 1 (Reprint In E. Benveniste (1966). Problèmes de linguistique générale, vol. 1 (pp. 237-250). Paris: Gallimard).

Bertinetto, P.M. (1990). Perifrasi verbali italiane: criteri di identificazione e gerarchia di perifrasticità. In G. Bernini \& A.G. Ramat (Eds.), La temporalità nell'acquisizione di lingue seconde (pp. 331-350). Milano: Franco Angeli.

Bertinetto, P.M. (1991). Il verbo. In L. Renzi, G. Salvi \& A. Cardinaletti (Eds.), Grande grammatica italiana di consultazione, vol. 2 (pp. 13-161). Bologna: Il Mulino.

Bianco, F. (2013). Il cum inversum fra italiano antico e moderno. In E.C. Herrero \& C.C. Rigual (Eds.), Actes del $26^{e}$ Congrés de Lingüistica i Filologia Romàniques (Valencia, 6-11 de setembre de 2010) (pp. 3213-3224). Berlin: De Gruyter.

Blakemore, D. (1999). Evidence and Modality. In K. Brown \& J. Miller (Eds.), Concise Encyclopedia of Grammatical Categories (pp. 141-145). Amsterdam: Elsevier.

Coseriu, E. (1980). Aspect verbal ou aspects verbaux? Quelques questions de théorie et de méthode. In J. David \& R. Martin (Eds.), La notion d'aspect (pp. 13-25). Paris: Klincksieck.

Dardano, M., \& Trifone, P. (2009). Grammatica italiana. Con nozioni di linguistica. Bologna: Zanichelli.

Dik, S.C. (1989). The Theory of Functional Grammar, Part I: The Structure of the Clause, Dordrecht - Holland / Providence RI - USA: Foris publications.

Fici, F. (2008). La categoria dell'evidenziale nelle lingue slavo-balcaniche. In P.M. Bertinetto et al. (Eds.), Categoria del verbo. Diacronie, Teoria, Tipologia. Atti del XXXI Convegno della Società Italiana di Glottologia, Pisa, 26-28 ottobre 2006 (pp. 133-157). Roma: Il Calamo. 
Gosselin, L. (2011). L'aspect de phase en français : le rôle des périphrases verbales. Journal of French Language Studies, 21(2), 149-171.

Guentchéva, Z. (1994). Manifestations de la catégorie du médiatif dans les temps du français. Langue française, 102, 8-23.

Holvoet, A. (2012). Polish mieć and the semantic map of interpretive deontics. Zeitschrift für Slawistik, 57(2), 129-146.

Jakobson, R. (1963). Les embrayeurs, les catégories verbales et le verbe russe. In R. Jakobson, Essais de linguistique générale, vol. I (pp. 176-196). Paris: Editions de Minuit.

Jansen, H. (2010). Verbi fraseologici. In Enciclopedia dell' Italiano. Roma: Treccani.

Le Draoulec, A. (2003). Quand, jusqu'à ce que et avant que : quelques cas particuliers de subordination temporelle hors présupposition. In J. Draghicescu (Ed.), Dix ans de Séminaire de Didactique Universitaire - Recueil anniversaire (pp. 175-196). Craiova: Editura Universitaria Craiova.

Le Draoulec, A. (2006). De la subordination à la connexion temporelle. $\mathrm{Ca}$ hiers Chronos, 15, 39-62.

Lempp, A. (1986). Mieć. 'To have' in modern Polish. München: Verlag Otto Sagner.

Lyons, J. (1989). Semantyka 2. Warszawa: PWN.

Nowakowska, M. (2017). Interpretacja prospektywna peryfrazy <mieć + bezokolicznik>. Biuletyn Polskiego Towarzystwa Językoznawczego, VXXIII, 221-246.

Nowakowska, M. (2018). La necessità di distinguere la perfettività dalla risultatività per comprendere il funzionamento dei tempi verbali italiani e polacchi. Italica Wratislaviensia, 9(1), 159-183.

Nowakowska, M. (2019). Entre médiativité et modalité. Analyse de la périphrase polonaise «mieć (avoir) + infinitif » et ses correspondants français. In O. Inkova \& D. Mancheva (Eds), Contrastes. Etudes de linguistique slavo-romane (pp. 1-22). Alessandria: Edizioni dell'Orso.

Reichenbach, H. (1947). Elements of symbolic logic. New York: The Free Press.

Renzi, L. (1987). Nuova introduzione alla filologia romanza. Bologna: Il Mulino.

Salvi, G., \& Vanelli, L. (2004). La nuova Grammatica italiana. Bologna: Il Mulino.

Squartini, M. (1998). Verbal periphrases in Romance. Aspect, actionality and grammaticalization. Berlin/New York: Mouton de Gruyter. 
Squartini, M. (2003). Disentangling evidentiality and epistemic modality in Romance. Lingua, 114, 873-895.

Strudsholm, E. (1999). Perifrasi verbali ed altri modificatori del processo verbale. In H.P. Lund (Ed.), Etudes Romanes 44 (La langue, les signes et les êtres : actes du colloque de l'Institut d'Études Romanes de l'Universié de Copenhague, le 3 octobre 1998) (pp. 47-60). Copenhagen: Museum Tusculanum Press.

Topolińska, Z. (1968). Miejsce konstrukcji z czasownikiem mieć w polskim systemie werbalnym. Studia Orientalis, 17(3), 427-431 (reprint in Z. Topolińska (2008). Z Polski do Macedonii, vol 1 (pp. 24-29). Kraków: LEXIS).

Topolińska, Z. (2000). „Dystans” - informacja zgramatykalizowane w polskim systemie werbalnym? In J. Mindak \& K. Wrocławski (Eds.), Folia Philologica Macedono-Polonica, tom 5 (pp. 86-93). Warszawa: UW (reprint in Z. Topolińska (2008). Z Polski do Macedonii, vol 1 (pp. 286-292). Kraków: LeXIS).

\footnotetext{
Riassunto: La perifrasi verbale stare per + INFINITO esprime l'aspetto prospettivo. Questo significato aspettuale può esser definito nei termini della teoria di Reichenbach (1947), che prevede l'utilizzo di tre momenti: MA (momento dell'avvenimento), ME (momento dell'enunciazione) e MR (momento di riferimento). Il significato prospettivo appare quando il MR precede il MA e quando la posizione del ME non ha importanza. L'autore di questo articolo esamina i possibili modi di tradurre in polacco la perifrasi verbale italiana stare per + INFINITO. A differenza dell'italiano, questa lingua slava non ha grammemi che convoglino il significato prospettivo. Tra le possibili traduzioni, la perifrasi verbale $<$ mieć (avere) + INFINITO> sembra esprimere al meglio il significato prospettivo. Inoltre, questa perifrasi verbale polacca ha altri due significati: modale ed evidenziale.
}

Parole chiave: aspetto prospettivo, aspetto imminenziale, italiano, polacco, stare per + INFINITO, mieć + INFINITO. 